\title{
Near miss na atenção primária à saúde e a segurança do paciente: revisão integrativa
}

RESUMO | Objetivo: Identificar as evidências sobre os incidentes near miss em serviços de atenção primária à saúde. Método: Revisão integrativa de literatura a partir de artigos primários publicados em sete bases de dados. Utilizou-se os descritores "near miss", "atenção primária à saúde" e "gestão da segurança" sem limitações quanto ao ano de publicação e idiomas. Resultados: Os tipos de incidentes near miss notificados com maior frequência estavam relacionados a erros de medicação, com variação entre 6,2\% e 96\%, e o processo de prescrição foi o mais recorrente. Os profissionais de saúde foram os responsáveis por interceptar entre $66 \%$ a $83 \%$ dos incidentes. Conclusão: A notificação de incidentes near miss deve ser incentivada e incorporada nas práticas gerenciais. Conhecer precocemente os erros e seu potencial de dano possibilita ações de melhorias para segurança do paciente.

Palavras-chaves: Near miss; Segurança do paciente; Atenção primária a saúde.

ABSTRACT | Objective: To identify the evidence about near miss incidents in primary health care services. Method: Integrative literature review based on primary articles published in seven databases. The descriptors "near miss", "primary health care" and "safety management" were used without limitations regarding the year of publication and languages. Results: The types of near miss incidents most frequently reported were related to medication errors, ranging from $6.2 \%$ to $96 \%$, and the prescription process was the most recurrent. Health professionals were responsible for intercepting between $66 \%$ to $83 \%$ of incidents. Conclusion: Reporting of near miss incidents should be encouraged and incorporated into management practices. Knowing the errors early and their potential for damage enables improvement actions for patient safety.

Keywords: Near miss, healthcare; Patient safety; Primary health care.

RESUMEN | Objetivo: identificar la evidencia sobre incidentes near miss en los servicios de atención primaria de salud. Método: revisión bibliográfica basada en artículos primarios publicados en siete bases de datos. Los descriptores "near miss salud", "atención primaria de salud" y "gestión de seguridad" se utilizaron sin limitaciones con respecto al año de publicación y los idiomas. Resultados: los tipos de incidentes cercanos a fallas más frecuentes se relacionaron con errores de medicación, que oscilaron entre $6.2 \%$ y $96 \%$, y el proceso de prescripción fue el más recurrente. Los profesionales de la salud fueron responsables de interceptar entre el $66 \%$ y el $83 \%$ de los incidentes. Conclusión: Se debe alentar e incorporar a las prácticas de gestión la notificación de incidentes cercanos. Conocer los errores temprano y su potencial de daño permite acciones de mejora para la seguridad del paciente.

Palabras claves: Near miss salud; Seguridad del paciente; Atención primaria de salud.

\section{Meire Augusta Celestino Amaro}

Enfermeira. Mestre em Ciências da Saúde pela Universidade Federal de São Paulo. Enfermeira de Auditoria de Processos do Hospital Israelita Albert Einstein.

ORCID: 0000-0002-4202-5500

\section{Mariana Cabral Schveitzer}

Enfermeira. Pós-doutora pelo Departamento de Orientação Profissional da Escola de Enfermagem da Universidade de São Paulo. Professora Adjunta do Departamento de Medicina Preventiva da Escola Paulista de Medicina da Universidade Federal de São Paulo.

ORCID: 0000-0001-9833-2932

\section{Elena Bohomol}

Enfermeira. Livre-Docente pelo Departamento de Orientação Profissional da Escola de Enfermagem da Universidade de São Paulo. Professora associada pelo Departamento de Administração em Serviços de Saúde e Enfermagem da Escola Paulista de Enfermagem da Universidade Federal de São Paulo.

ORCID: 0000-0002-7196-0266
Recebido em: 08/07/2020

Aprovado em: 17/12/2020

INTRODUÇÃO

ncidentes em serviços de saúde são problemas investigados mundialmente devido à capacidade de causar danos aos pacientes e mitigá-los se tornou um grande desafio para as organizações $^{(1)}$. Destaca-se a importância de notificar os eventos adversos para que as melhorias nas instituições de saúde sejam implementadas.

No entanto, a notificação tanto de eventos adversos como incidentes near miss, entendidos como quase erro devido à detecção da falha antes de atingir o paciente, não é foco de atenção dos gestores e, ainda, pouco se valoriza este tipo de notificação ${ }^{(2)}$. Sabe-se que possuem procedência semelhante sendo necessá- ria a análise de sua causa raiz, para que medidas preventivas sejam instauradas antes que atinjam os pacientes ${ }^{(3-5)}$.

No Brasil, a obrigatoriedade da notificação de incidentes por todos os serviços de saúde foi instituída por meio da Resolução de Diretoria Colegiada (RDC) $\mathrm{n}^{\circ}$ 36/2013, com a implantação dos núcleos de segurança do paciente ${ }^{(6)}$. A instituições hospitalares representam mais de $90 \%$ dos serviços de saúde que cadastraram seus núcleos na Agência Nacional de Vigilância Sanitária (Anvisa) e as unidades básicas de saúde apresentam baixa adesão para a realização de notificações, representando $1 \%$ dos serviços cadastrados ${ }^{(7)}$.

A atenção primária à saúde (APS) é a porta de entrada do paciente no sistema de saúde e requer o monitoramento da qualidade e segurança dos serviços que dispensa. Conhecer as consequências dos eventos adversos e as razões dos in- 
cidentes near miss pode auxiliar na busca de um atendimento seguro ${ }^{(8,9)}$.

Estudos sobre incidentes near miss na APS representam uma lacuna do conhecimento e estudá-los pode acrescentar maior valor à qualidade em razão da inexistência do dano ao paciente e ausência do sentimento da culpa ou risco de litígio ${ }^{(10,11)}$.

O objetivo deste estudo é identificar as evidências sobre os incidentes near miss em serviços de APS.

\section{MÉTODO}

Revisão integrativa composta pelas seguintes etapas: identificação da questão do estudo; identificação dos estudos relevantes; seleção dos estudos; extração dos dados de interesse; organização dos dados e apresentação dos resultados ${ }^{(12)}$.

O estudo mapeou as investigações na APS para responder a seguinte questão: Quais são os tipos de incidentes near miss notificados nos serviços de APS? Utilizou-se o acrômio PICo, onde o "P" (População) incluiu os serviços que reportam incidentes near miss, o "I" (Intervenção) foi a notificação de near miss e o "Co" (Contexto) relativo aos serviços de APS.

A busca foi realizada nas seguintes bases de dados: National Library of Medicine and National Institutes of Health (PubMed), Web of Science, Literatura Latino-Americana e do Caribe em Ciências da Saúde (LILACS), SCOPUS, Cumulative Index to Nursing and Allied Health Literature (CINAHL), EMBASE e Base de Dados de Enfermagem (BDENF). Os descritores e suas combinações foram baseados nos vocabulários controlados DeCS (Descritores em Ciências da Saúde) e MESH (Medical Subject Headings). A estratégia de busca foi: (Gestão da segurança OR Safety management OR gestión de la seguridade) AND (Quase erro OR Near miss OR Near miss healthcare OR Near miss salud OR Notificação de incidentes OR Incident reporting e Notificación de incidentes) AND ( Atenção primária à saúde OR Primary healthcare OR Atención primaria de salud).

\section{As buscas} resultaram em um total de 472 artigos e oito estudos atenderam aos critérios

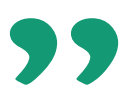

A coleta de dados ocorreu em janeiro a maio de 2019 e houve mais uma atualização em agosto 2019. Os critérios de inclusão foram os artigos originais, que investigaram incidentes near miss em serviços de APS, sem limitações de ano de publicação e idiomas. Foram critérios de exclusão os que não apresentaram informações sobre incidentes near miss nos resultados.

O levantamento de dados foi conduzido por dois pesquisadores, de forma independente. Para seleção dos estudos, foi realizada a leitura do título e resumo, respectivamente. Os dados extraídos dos artigos foram resumidos e organizados em uma planilha da Microsoft Excel $\AA$, com seguintes itens: Identificação autor e ano do estudo, objetivos, resultados e conclusões.

\section{RESULTADOS}

As buscas resultaram em um total de 472 artigos e oito estudos atenderam aos critérios, conforme mostra a Figura 1.

A Figura 2 resume as principais características dos artigos incluídos na revisão, observando-se maior frequência de publicações no ano de 2007 (14-16). As publicações norte-americanas são a maioria ${ }^{(14,17-20)}$ e não foram identificados estudos brasileiros.

Verificou-se que quatro avaliaram sistemas de notificação de incidentes e os tipos de incidentes notificados ${ }^{(17,19-21)}$. A maioria investigou os incidentes de um modo geral,
Figura 1. Diagrama do processo de seleção dos estudos. São Paulo, SP, Brasil, 2019. Adaptado do PRISMA-ScR ${ }^{(13)}$.
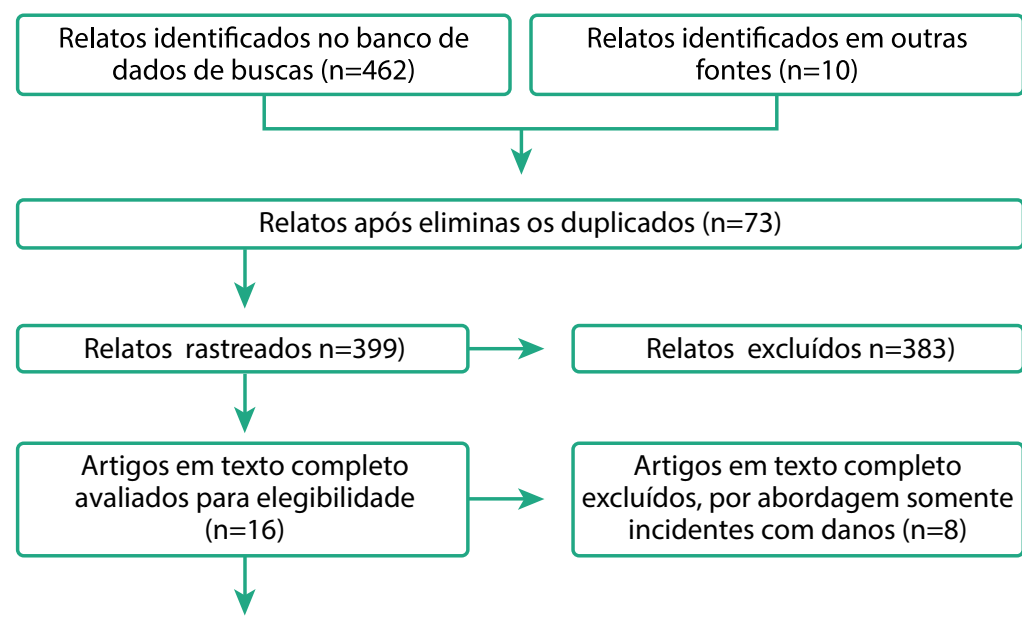

Relatos excluídos $\mathrm{n}=383$ )

Artigos em texto completo excluídos, por abordagem somente incidentes com danos $(n=8)$

Estudos incluídos na revisão $(n=8)$ 
sendo apontada a variação de $0,5 \%$ a $86 \%$ de notificação de near miss ${ }^{(15-18,21)}$.

Dos tipos de incidentes near miss encontrados cinco estudos apontaram para os erros de medicação, com uma variação de $6 \%$ a $96 \%$ sendo os erros com prescrição o de maior recorrência ${ }^{(15,17-19,21)}$.
Os estudos que analisaram a interrupção de erros nos serviços de APS revelaram que a detecção era feita pelos profissionais enfermeiros, farmacêuticos e médicos e variaram entre $66 \%$ a $83 \%$. Os pacientes representaram de 15\% a $17 \%$ das barreiras que impediram os incidentes $^{(14,18)}$.
Diante da potencial gravidade dos incidentes, foram identificados como fatores contribuintes a sobrecarga de trabalho e a fragmentação da assistência ${ }^{(15,16,18)}$.

Quanto às características dos sistemas de notificação investigados, a maioria apontou para a necessidade do anonimato para quem faz o registro dos incidentes ${ }^{(17,19-21)}$.

\section{Figura 2. Sintese dos oito artigos sobre incidentes near miss na atenção primária à saúde. São Paulo, SP, Brasil, 2019.}

\section{Autor}

Objetivos

Investigar a viabilidade de um sistema de auto relato para erros de disChua et al. (21), pensação e near miss em farmácias 2003. comunitárias. Identificar os tipos de erros ou near miss presentes em farmácias comunitárias.

Parnes et al. ${ }^{(14)}$, 2007.

Examinar como as cascatas de erros são interrompidas antes de afetarem os pacientes.

\section{Resultados}

De um total de 51.357 itens dispensados, foram detectados $247(0,5 \%)$ near miss. Os incidentes near miss ocorreram seis vezes mais do que erros de dispensação, indicando a importância da verificação final nas farmácias.

Dos 754 eventos notificados, $8 \%$ eram near miss. Médicos, enfermeiros e farmacêuticos interromperam $83 \%$ dos erros. Pacientes e familiares interromperam 15\% dos erros.

Knudsen et Descrever a frequência e gravidade al. ${ }^{(15)}, 2007 . \quad$ dos erros registrados em farmácias comunitárias.
Foram notificados 1.015 eventos e $23 \%$

foram near miss. Os erros de prescrição foram os mais frequentes $(96 \%)$.

\section{Conclusões}

0 auto relato anônimo de incidentes é prático e viável. Os incidentes near miss mais comum foram: a forma incorreta da medicação, medicamentos incorretos, quantidade incorreta e dosagem incorreta.

Destaca a importância das pessoas na detecção e correção de erros. Reforça a necessidade de capacitação profissional para ações proativas diante de erros.

Os erros de prescrição foram os mais frequentes com potencial de causar danos ao paciente. 0 número de erros de medicação é alto devido ao grande volume de prescrições geradas em serviços de APS na Dinamarca.

Foram classificados 71 incidentes, sendo $70 \%$ near miss e $30 \%$ eventos adver-

sos. Os fatores contribuintes foram a e Delaney ${ }^{(16)}$ com potencial de danos a pacientes 2007. de cuidados primários.

sobrecarga de trabalho e fragmentação da atividade.

Descrever o tipo e a evitabilidade potencial de erros de medicação

Kuo et.al. ${ }^{(18)}$, 2008. médicos de família utilizando uma ferramenta de codificação de erro de medicação. relatados em consultórios de

Dos 178 erros de medicação identificados, $40 \%$ eram near miss. As principais categorias profissionais que impediram o incidente foram: farmacêuticos ( $40 \%$ ) e médicos (19\%). Os achados mostraram que $17 \%$ dos incidentes foram relatados pelos pacientes.
Kennedy et al. ${ }^{(17)}, 2008$.

Implementar um sistema de notificação de erros de prescrição em consultórios de cuidados primários.
Dos 165 incidentes analisados $86 \%$ eram near miss e $12 \%$ incidentes sem danos.
Os erros de prescrição foram os mais frequentes. 0 uso de ferramentas eletrônicas é um bom recurso para conhecer o potencial de erros.

Enfermeiros e funcionários de consultórios foram considerados um recurso valioso para relatar erros de prescrição. Ações permanentes para estimular e sustentar o sistema de notificação.
Foram notificados 632 near miss. Os tipos mais comuns foram $47 \%$ falhas nos processos administrativos; $25 \%$ erros de arquivamento; $15 \%$ erros de lançamento de dados. Os erros relacionados ao processo de medicação foram: $7 \%$ ao processo

de administração de medicamentos e $6 \%$ ao de prescrição.
Crane et al. ${ }^{(19)}$, 2015.
Analisar a viabilidade de relatos regulares de incidentes near miss $\mathrm{e}$ investigar os tipos mais frequentes.
A notificação anônima dos incidentes near miss foi bem sucedida em serviços de APS. Os eventos mais frequentes foram as falhas administrativas

Mesmo com alto índice de near miss os eventos adversos graves também foram relatados. As falhas, estavam relacionadas a danos potencialmente graves que pudessem atingir o paciente. 


\author{
Identificar as barreiras e aspectos \\ Crane et al.(20), facilitadores da implementação de \\ 2017.
}

Nenhuma barreira foi identificada para implementação do sistema de relatos de near miss. Foram identificados 34 projetos de melhorias.
A notificação anônima contribuiu para o sucesso deste recurso. Cabe à liderança manter o compromisso incentivar as notificações.

\section{DISCUSSÃO}

A presente pesquisa confirma a lacuna de conhecimento em relação aos incidentes near miss, constatando a escassez de estudos que investigam este tema na APS que pode estar relacionada à dificuldade de entendimento em seu conceito e à prioridade dada aos incidentes com dano. A definição do que realmente caracteriza um near miss nem sempre é entendida pelos profissionais gerando a subnotificação deste tipo de erro ${ }^{(22)}$.

Estudos sobre near miss apontam que há falta de sensibilidade por parte da liderança dos serviços para a prevenção do dano. No entanto, sobressai o olhar para a gestão do dano, isto é, os eventos que atingiram o paciente ${ }^{(23)}$. Todavia, analisar os incidentes near miss identifica a chance de sua recorrência e a oportunidade de aprender com eles. Estudo destaca que a cada 600 near miss identificados, 30 podem se tornar incidentes sem danos, 10 podem se tornar incidentes com danos e um pode se tornar incidente com lesão grave ou morte ${ }^{(24)}$.

A pesquisa também revelou que o Sistema de Notificação de Incidentes (SNI) é a ferramenta utilizada para conhecer os erros ressaltando para a importância de se ter dados e eles se transformarem em boas informações para gerar ações de mudança para as práticas do cuidado ${ }^{(4,25)}$.

Todavia, o uso do SNI ainda apresenta barreiras em diferentes ambientes e no aspecto relacionado aos incidentes near miss elas não são diferentes. As justificativas para subnotificação dos incidentes referem para o aumento da sobrecarga de documentos que os profissionais devem preencher e da ausência do prejuízo ao paciente, perpetuando o comportamento
Os incidentes near miss relacionados à medicação esttavam mais presentes no processo de prescrição, apontando para a necessidade de dar especial atenção ao profissional prescritor. de "corrigir e esquecer" e não o de "consertar e informar"(22).

Os tipos de near miss estavam relacionados aos erros de medicação. Estes são erros frequentes nos serviços de saúde, incluindo a APS. A pesquisa apresentou uma variação ampla, explicada pelas opções metodológicas e os objetivos dos estudos realizados, uma vez que os desenhos, amostra, procedimentos de coleta de dados não foram uniformes ${ }^{(15-18,21)}$.

Os incidentes near miss relacionados à medicação esttavam mais presentes no processo de prescrição, apontando para a necessidade de dar especial atenção ao profissional prescritor. Para redução de erros de medicação há de se pensar em estratégias como educar os profissionais de saúde e os pacientes, implementar a conciliação medicamentosa e utilizar sistemas informatizados ${ }^{(26)}$.

Embora seja uma situação incomum o erro resultante dos processos administrativos, eles também pode atingir o paciente. Confirmam a afirmativa diferentes estudos sobre eventos adversos em cuidados primários que apresentam como causa dos erros as atividades referentes aos processos administrativos, como erro de agendamento, de preenchimento, registros incompletos, dentre outros ${ }^{(22,25)}$.

Destaca-se o papel dos profissionais enfermeiros e farmacêuticos em identificar falhas precocemente. Eles são barreiras na prevenção de incidentes, cabendo ao primeiro desempenhar o papel de líder da equipe assistencial e, ao segundo, a participação na segurança de todo o processo de medicação ${ }^{(27,28)}$.

Além disto, o paciente é um importante aliado para sua própria segurança contribuindo na detecção dos erros, portanto deve ser educado e estimula- 
do a participar das decisões sobre o seu cuidado $^{(27)}$.

\section{CONCLUSÃO}

Os erros de medicação foram os mais prevalentes e o processo de prescrição foi considerado o mais frágil dentro do sistema. Verificou-se a importância dos profissionais e pacientes estarem preparados para detecção precoce de incidentes e, desta forma, atuarem como barreiras efetivas. A notifica- ção do incidente near miss deve ser incentivada e incorporada nas práticas de notificação pois ao se conhecer precocemente os erros e seu potencial de dano amplia a possibilidade de implementar ações de meIhorias para segurança do paciente. 1

\section{Referências}

1. Siman AG, Brito MJM, Mudanças na prática de enfermagem para melhorar a segurança do paciente. Rev Gaúcha Enferm. [Internet]. 2016 [cited 2020 July 04] ; 37 ( spe ): e68271. Available from: http://www.scielo.br/scielo.php?scrip$\mathrm{t}=$ sci_arttext\&pid=\$1983-14472016000500413\&lng=en. Epub Apr 27, 2017. doi: https://doi.org/10.1590/1983-1447.2016.esp.68271.

2. World Health Organization. The Conceptual Framework for the International Classification for Patient Safety Version 1.1. Genebra:WHO; 2009. 154 p. Final Technical Report. [Cited 2019 Jul 23]. Available https://www.who.int/patientsafety/taxonomy/icps_full_report.pdf.

3. Carter AW, Mossialos E, Darzi A. A national incident reporting and learning system in England and Wales, but at what cost? Expert Rev Pharmacoecon Outcomes Res. 2015;15(3):365-8. doi: 10.1586/14737167.2015.1031114.

4. Van Spall H, Kassam A, Tollefson TT. Near-misses are an opportunity to improve patient safety. Curr Opin Otolaryngol Head Neck Surg. 2015;23(4):2926. doi: 10.1097/M00.0000000000000177.

5. Silva APF, Backes DS, Magnago TSBS, Colomé JS. Segurança do paciente na atenção primária: concepções de enfermeiras da estratégia de saúde da família. Rev Gaúcha Enferm. 2019;40(esp):e20180164. doi: https://doi. org/10.1590/1983-1447.2019.20180164.

6. Brasil. Ministério da Saúde. Agência Nacional de Vigilância Sanitária. Resolução da Diretoria Colegiada - RDC no. 36, de 25 de julho de 2013. Institui ações para a segurança do paciente em serviços de saúde e dá outras providências. Diário Oficial da União 2013. [Citado 2019 Jul 03]. Disponível em: https://www20.anvisa.gov.br/segurancadopaciente/index.php/legislacao/ item/rdc-36-de-25-de-julho-de-2014.

7. Brasil. Ministério da Saúde. Agência Nacional de Vigilância Sanitária. Boletim Segurança do Paciente e Qualidade em Serviços de Saúde $n^{\circ} 18$ : Incidentes relacionados à assistência à saúde 2017. Brasilia: GVIMS/GGTES/ANVISA; 2018. [Citado 2020 Jun 03]. Disponível em: file:///C:/Users/User/Downloads/ BOLETIM_2017_INCIDENTES_EA.pdf.

8. Astier-Peña MP, Torijano-Casalengua M, Olivera-Cañadas G. Prioridades en seguridad del paciente en Atención Primaria. Aten Primaria. 2015;48(1):3-7. doi: 10.1016/j.aprim.2015.08.001.

9. Panesar SS, Silva D, Carson-Stevens A, Cresswell KM, Salvilla SA, Slight SP, Javad S. How safe is primary care? A systematic review. BMJ Qual Saf. 2015;25(7):544-53. doi: 10.1136/bmjqs-2015-004178.

10. Barach P, Small SD. Reporting and preventing medical mishaps: lessons form non-medical near miss reporting. BMJ. 2000;320:759-63. doi: https://doi. org/10.1136/bmj.320.7237.759.

11. World Health Organization. Draft guidelines for adverse event reporting and learning systems: from information to action. Genebra:WHO; 2005. [Cited 2020 Jun 23] Available from: https://www.jeder-fehler-zaehlt.de/lit/further/Reporting_Guidelines.pdf..

12. Sousa LMM, Marques-Vieira CMA, Severino SSP, Antunes AV. A metodologia de revisão integrativa da literatura em enfermagem. RIE. 2017:17-26 [Citado 2020 Jul 02]. Disponível em: https://www.researchgate.net/publication/321319742_Metodologia_de_Revisao_Integrativa_da_Literatura_em_ Enfermagem

13. Tricco AC, Lillie E, Zarin W, O'Brien KK, Colquhoun $H$, Levac D, et al. PRISMA extension for scoping reviews (PRISMA-ScR): Checklist and explanation. Ann Intern Med. 2018;169(7):467-73. doi: https://doi.org/10.7326/M18-0850.

14. Parnes B, Fernald D, Quintela J, Araya-Guerra R, Westfall J, Harris D et al. Stopping the error cascade: A report on ameliorators from the ASIPS collaborative. Qual Saf Heal Care. 2007;16(1):12-6. doi: https://doi.org/10.1136/ qshc.2005.017269.

15. Knudsen P, Herborg H, Mortensen AR, Knudsen M, Hellebek A. Preventing medication errors in community pharmacy: Frequency and seriousness of medication errors. Qual Saf Heal Care. 2007;16(4):291-6. doil: https://dx.doi. org/10.1136\%2Fqshc.2006.018770.

16. Kostopoulou O, Delaney B. Confidential reporting of patient safety events in primary care: Results from a multilevel classification of cognitive and system factors. Qual Saf Heal Care. 2007;16(2):95-100. doi: https://doi.org/10.1136/ qshc.2006.020909.

17. Kennedy AG, Littenberg B, Senders JW. Using nurses and office staff to report prescribing errors in primary care. Int J Qual Heal Care. 2008;20(4):23845. doi: https://dx.doi.org/10.1093\%2Fintqhc\%2Fmzn015.

18. Kuo GM, Phillips RL, Graham D, Hickner JM. Medication errors reported by US family physicians and their office staff. Qual Saf Health Care. 2008;17(4): 286-90. doi:10.1136/qshc.2007.024869.

19. Crane S, Sloane PD, Elder NC, Cohen L, Laughtenschlaeger N, Walsh K, et al. Reporting and using near-miss events to improve patient safety in diverse primary care practices: A collaborative approach to learning from our mistakes. J Am Board Fam Med. 2015;28(4):452-60. doi: https://doi.org/10.3122/ jabfm.2015.04.140050.

20. Crane S, Sloane PD, Elder NC, Cohen LW, Laughtenschlager N, Zimmerman S. Implementing near-miss reporting and improvement tracking in primary care practices: Lessons learned. [Cited 2020 .Jun 20]. Available from: https:// www.ahrq.gov/patient-safety/resources/liability/crane.html

21. Chua SS, Wong ICK, Edmondson H, Allen C, Chow J, Peacham J, et al. A feasibility study for recording of dispensing errors and "near misses" in four UK primary care pharmacies. Drug Saf. 2003;26(11):803-13. doi: https://doi. org/10.2165/00002018-200326110-00005.

22. Hewitt TA, Chreim S. Fix and forget or fix and report: a qualitative study of tensions at the front line of incident reporting. BMJ Qual Saf. 2015;24:303-10. doi: 10.1136/bmjqs-2014-003279.

23. Rees P, Edwards A, Panesar S, Powell C, Carter B, Williams H, Carson-Stevens A. Safety incidents in the primary care office setting. Pediatrics. 2015;135(6):1027-35. doi: https://doi.org/10.1542/peds.2014-3259.

24. Cure L, Zayas-Castro J, Fabri P. Clustering-based methodology for analyzing near-miss reports and identifying risks in healthcare delivery. J Biomed Inform. 2011;44(5):738-48. doi: 10.1016/j.jbi.2011.03.012.

25. Silva AS, Maciel GA, Wanderley LSL, Wanderley AG. Indicadores do uso de medicamentos na atenção primária de saúde: uma revisão sistemática. Rev Panam Salud Publica. 2017;41:132. doi: 10.26633/ RPSP.2017.132

26. Santos PRA, Rocha FLR, Sampaio CSJC. Ações para segurança na prescrição, uso e administração de medicamentos em unidades de pronto atendimento. Rev Gaúcha Enferm. 2019;40(esp):e20180347.doi: https://doi. org/10.1590/1983-1447.2019.20180347.

27. Alves MFT, Carvalho DS, Albuquerque GSC. Barriers to patient safety incident reporting by Brazilian health professionals: an integrative review. Ciênc saúde coletiva [Internet]. 2019 Aug [cited 2020 July 04] ; 24( 8 ): 2895908. Available from: http://www.scielo.br/scielo.php?script=sci_arttext\&pi$\mathrm{d}=\mathrm{S} 1413-81232019000802895 \& \mathrm{lng}=e n$. Epub Aug 05, 2019. http://dx.doi. org/10.1590/1413-81232018248.23912017.

28. Cunha DC, Carvalho DNR, Batista AMV, Santos APG, Martins JDN, Souza MOLS. A percepção da equipe de enfermagem sobre a importância da segurança do paciente em um hospital público do Norte. Nursing, 2020; 23(260):3512-15. 